\title{
Development and Psychometric Attributes of a Theory-Based Physical Activity Assessment Questionnaire for the Pregnant Women (PA2Q-PW)
}

Leila Kianfard ( $\sim$ leila_kianfard@madares.ac.ir)

Tarbiat Modares University Faculty of Medical Sciences https://orcid.org/0000-0002-7657-732X

Farkhonde Amin SHokravi

Tarbiat Modares University Faculty of Medical Sciences

Sakineh Rakhshanderou

Shahid Beheshti University of Medical Sciences School of Public Health and Safety

Shamsaddin Niknami

Tarbiat Modares University Faculty of Medical Sciences

\section{Research}

Keywords: physical activity, psychosocial determinants, pregnant women, questionnaires

Posted Date: January 15th, 2021

DOI: https://doi.org/10.21203/rs.3.rs-144609/v1

License: (c) (i) This work is licensed under a Creative Commons Attribution 4.0 International License.

Read Full License 


\section{Abstract}

\section{Background}

This study's goal was to design and validate questionnaires for using to distinguish PEN-3 cultural model determinant factors of exercise in pregnant women. A questionnaire of physical activity theory-based was performed in 400 pregnant women with the first pregnancy. The validation of questionnaire design was used to develop the measures, including validity, reliability, and factor analysis by correlating scores to be an exercise of pregnant women during pregnancy.

\section{Methods}

The perception scale contained two factors: Knowledge and attitudes The Nurtures scale contained one element: Support was sought. The Enabling level included one element: barriers. Coefficients of reliability ranged from within 0.99. Among the four scores, significant correlations were achieved in this study. Four scales to measure PEN-3 cultural model determinants factors of being active during pregnancy were reformed for utilization with country, mostly Tehran-Iran, pregnant women, and shown to be validity and reliability.

\section{Results}

Factor analysis resulted in interpretable subclass that may be used as variables. These preliminary results provide support; for using the scales to measure the influences on pregnant women's physical activity.

\section{Conclusions}

The Physical Activity Questionnaire for pregnant women Patients (PA2Q-PW) is the first instrument that uses the PEN-3 cultural model within its constructs. The results indicated that PA2Q-PW is a valid and reliable instrument for assessing physical activity perceptions and is now existing and can be used in future studies.

\section{Background}

Pregnancy is a perfect moment with a pregnant woman and the life of her unborn infant. Then it is astonishing that maternal health is a health priority in the world [1]. There is evidence that physical activity plays an essential role in preventing illnesses like preeclampsia, type 2 diabetes, obesity, and many cancers [2]. Physical activity during pregnancy has further advantages, such as a diminished risk of HELP-syndrome, gestational diabetes mellitus, and additional gestational weight gain [3]. This could be individually significant for pregnant women that are from low and middle- income countries (LMICs), where low socioeconomic status (SES) in transitioning circumference, has been presented as a risk factor for numerous outbreak of lack of physical activity, overweight, GDM, and impaired glucose intolerance [4, 5]. As the same way, in the United States of America it is introduced that in lack of obstetric 
complications, as per the guidelines of adults, pregnant women must accumulate 30 minutes or more of moderate-severe physical activity on most, and preferred all of the days in a week [6, 7]. The American College of Obstetrics and Gynecology (ACOG) introduced that pregnant women employ $30 \mathrm{~min}$ of having physical activity a minimum of 5 days per week [8].

Along with dieting, being an activity during pregnancy maintains promise as a natural, impressive intervention to repair infant and maternal safety consequences [9-10]. Howsoever, some pregnant women seem to follow and chase the guidelines [11]. Meanwhile, the study in Iran showed that $70 \%$ of pregnant women did not have any physical activity [12]. Having activity is one of the substantial factors affecting the quality of life. Currently, it is increasingly specified that the assessment and consideration of the quality of life make essential data in connection with the description of the health situation in various groups [13]. Among these, we can mention cases such as reducing the incidence of HTN and preeclampsia. Regular exercise in the first 20 weeks of pregnancy reduces the venture of preeclampsia by up to $34 \%[14,15]$. One of the approaches to increasing lifetime activity is to use theoretical research, or In other words, the theory-based to recognize influences on activity behavior during pregnancy. The lack of success about many exercise promotion endeavors may be due to the lack of reconnaissance of theory-based determinants of health of activity, resulting and inferencing in inappropriate and unsuitable program strategies and content [16]. The Theory exerted in a physical activity determinants study among pregnant women is the PEN-3 cultural model [17]. Perceptions factors in one's capability to be prosperous at being active is a correlate of activities among the groups of both youth and adults [18-20].

Physical activity in pregnancy may not be permanent in the trimester of pregnancy, because of quick variation in the pregnant woman's body. Therefore, it may be valuable to collect Theory based-on cultural model determinants in a period of physical activity during pregnancy. In this study, we developed a questionnaire to evaluate psychosocial and perceptual determinants based on the PEN-3 cultural model about different physical activity levels in pregnancy for utilization in the two and third periods of pregnancy (PA2Q-PW). It was developed so that response could be probed for alteration in physical activity in a shorter time. Nevertheless, there are some theoretical derivative tools to measure determinant factors of being active among pregnant women presented in the literature [21]. The purpose of doing this study was to develop and design a valuable questionnaire for physical activity in pregnant women and simultaneous pertaining validity for the $\mathrm{PA} 2 \mathrm{Q}$ organized questionnaire of physical activity among nulliparous pregnant women. A better understanding of basing-on cultural model permeations on physical activity may require cooperation endeavors in health centers and society settings to cultivate lifetime activity. This survey aimed to develop questionnaires and its validity used to scale perceptions and determinant factors about psychosocial agents of physical activity and concurrent related validity for the PA2Q-PEN3 structured questionnaire about physical activity among nulliparous women in two and three trimesters during pregnancy. Inactive life is the leading reason for fatality, disability, and illness, with about 2 million deaths each year due to a lack of physical activity.

Now there are no general validated scales so that are measuring perceptions, nurtures, and enabling pregnant women concerning exercise during pregnancy. Thus, the facility could provide assistance to 
comprehend women's prospects and, in turn, plan general interventional schedules for pregnant women. In this study, any of the questionnaires was established in the setting of direct scales of the PEN-3 cultural model. The principal objective of this survey was to develop a tool in a framework. A review of past studies has a presentation that people who recognize themselves as physical activity have more desirable intentions and employ considerably more in physical activity than people who do not [22, 23]. In the United States, $25 \%$ of people do not have any exercise in their workplace, and $12 \%$ of total mortality was attributed to low mobility [24] However, this study was limited to a group of pregnant women.

Meanwhile, the study in Iran showed that $70 \%$ of pregnant women did not have any physical activity [25]. Exercise is one of the significant factors affecting the quality of life. Currently, it is increasingly specified that measurement and assessment of the quality of life $(\mathrm{QOL})$ processed essential data in connection with the description of status about health in various groups [26]. Besides, this kind of study was limited to a group of nulliparous female pregnant women. The statistics and figures completely clear show that, like many other countries, pregnant women are suffering more from lack of activity in Iran. Further, the information from Iran shows that the outbreak of no mobility and inactivity lifestyle in males is less than that of females $[27,28]$. Sarrafzadegan et al. Realized that abdominal obesity was approximately six times as common in females as in males $(71.7 \%$ vs. $12 \%, P<0.05)[29]$.

\section{Material And Methods}

In this study, the entire data gathering designated herein was confirmed by the University of Tarbiat Modares - International Standard Randomized Controlled Trial. Every one of the pregnant women taken written understanding consent former to taking part in this study. We assessed the document for validity and simultaneous test-retest reliability from two independent groups of nulliparous women.

\section{The participants and study}

Because of the scale in a broad setting, a cross-sectional study was performed in health centers in Tehran, Iran, between January and March 2019. In this study, the inclusion criteria involved aged between 28 to 35 years, having literacy, have no background of severe complications of the disease, physical inactivity during pregnancy. To collect the data in this study, educated interviewers accomplished face-toface discussions and interviews. Reference number: Trial ID: ISRCTN13356179/

URL: https://doi.org/10.1186/ISRCTN13356179

\section{Data Collection}

The survey applied a sample of 400 pregnant women participating in a study for further validity in a period at the Tarbiat Modares University, Tehran. This group was pregnant women that were in two and three trimesters, and the pastoral age was 20 (M1/1420.30, SD1/43.93). The dissemination of racial for pregnant women was $97.2 \%$ of Asian and $2.8 \%$ minority. The determinants instruments (Perceptions, Nurtures, and Enabling) and the behavior (to be active) measure were managed as a section of the power of questionnaires and surveys with samples of concerning 400 pregnant women. In this survey, the 
questionnaire's moderator studies all the instructions and items to the center using standardized writing [30]. The writing contained a preface in which exercise was determined as "any kind of moving games, energetic play, sports, or physical activity that gains you acting, rapid breathing, and your heart racing faster." Advertising pictures demonstrating the range of conceivable exercise (e.g., swimming, dancing, running, and acting on the playground, biking, track and field, running, physical activities) companioned this presentation. The questionnaires were managed in the first course of the day whenever the midwives stayed in the health centers. Evaluations were accomplished anonymously, and nulliparous women had the choice to skip any item using the choice "I choose not to answer." Through each of the pregnant women, average (mean) scores were computed for every changeable presented $80 \%$ of the questions were responded on a defined subscale or scale. If a pregnant woman responded less than $80 \%$ of the questions, or items on a subscale or scale, afterward, a total score was not computed for that variable. Based on the information provided on these criteria, among $0 \%$ and $5 \%$ of nulliparous women were missing measure grants for particular variables (the scale of attitude was the most current missing variable findings from the $80 \%$ short cut). In this section, after data collection, the test-retest was chosen to develop the questionnaire with a proper subset of the identical sample $(n=45)$. Eventually, the testretest reliability questionnaires were managed with the identical protocol two weeks separate.

\section{PEN3 Physical Activity Questionnaire}

A 24-h summoned questionnaire was developed to assess exercise between nulliparous women in two and trimester registered in the PEN3 Survey. The questionnaire-based on PEN3 physical activity assessed perceptions, nurtures and enabling factors the woman participated in that to tend to lack of physical activity during pregnancy, including knowledge, attitude, social support, and environmental barriers, doctor's advice, wife and family consent, and both inward and out-of-door housekeeper processes which do not allow for physical activity. For example, the query requested: "I believe that with exercise, I can easily deal with problems such as gestational diabetes." For each activity in these questions, the participant reported the nurtures factors using the accordance options: 'strongly agree,' 'agree, 'neutral,' disagree,' strongly disagree.'

\section{Participants/Setting}

All 400 nulliparous pregnant women in five districts of Tehran in Iran (composed of nulliparous pregnant women in 21 categories) were asked to participate. Almost $85 \%$ of the people. In other words, 400 pregnant women participated with racial segregation of $89 \%$ Iranian-Asian and $9 \%$ Afghani-Asian (2\% unknown). In this case, Forty-five percent of participants were qualified free or diminished lunch. Finally, an aware satisfaction of pregnant women was received from all of them.

\section{Development of Questionnaire and Testing the Pilot}

The PEN-3 cultural model based on Theory provided the theoretical foundations for the instruments developed in this study. First, instruments previously used and tested with adults were identified. The perception scale was taken from the "beliefs about the consequences of participating in" physical activity 
from the "attitude toward the behavior" component of the PEN-3 cultural model [17]. This component also corresponds to barriers to physical activity in pregnant women. The Social Influences scale included items addressing the nurtures of others from the PEN-3 cultural model [17].

After conducting individual interviews and performing two focus group discussions with 40 pregnant women, the first questionnaire was designed based on the cultural PEN-3 model. Which includes the structures of perceptions, enablers, and nurtures, then primary concepts being measured, and sample items of final instruments are presented in Table 1.

The instruments went through a series of three pilot tests conducted in a health center setting with nulliparous pregnant women demographically similar to the study population. The initial pilot test indicated that pregnant women had difficulty understanding the wording of some items, tiny words for reading, and lengthy questionnaire sentences. Changes made to the primary instruments during the first pilot test included selecting short appropriate words and putting instruments in a visually more appealing format (giant print, more full margins). The subsequent two pilot tests refined and reduced the instruments' size by eliminating items that pregnant women did not understand. The administration time of the final instrument was about 20 min.

\section{Scale development}

The initial scale included of 41 items. These aspects included awareness of physical activity, attitude, nurtures, enabling, and barriers factors that effect on inactivity during pregnancy. Item response options in perceptional factors were on the Triploid scale (Yes, No, I do not know) also for nurtures and enabling factors as a 7-point scale from one (strongly disagree) to five (strongly agree) Table 1. Subsequently, a panel of 12 experts in the field of health education and health promotion, gynecologist, and physical activity inquired the original questionnaire. The expert was requested to the explanation on specific questions in connection to the resolution, clarification, and method. The items on the questionnaire were insignificancy modified according to panel reviews. Afterward, a various kind of 20 experts on health education and health promotion was requested that independently to the explanation of the relevance and necessity of the questions because of estimate respectively Content Validity Index (CVI) and Content Validity Ratio (CVR) in this study. For this purpose, the necessity of the questions was evaluated using a scale of three-point ranking, which is as follows: (i) not necessary, (ii) effective, but not necessary, and (iii) necessary. Conformity with the panel' evaluation, a Content Validity, Reliability (CVR) for all of the scales was calculated. As for Lawshe, if more than half of the panelists represent that each item is necessary, that question has a minimum of content validity [31]. In this study, the CVR for all of the Criteria was 0.82 , informing a satisfying outcome. The correlation of the questions was also evaluated, exerting a fourpoint of rating criteria: (i) no relevant, (ii) insignificancy relevant, (iii) relevant, and (iv) reliable relevant. The Content Validity Ratio (CVI) of every item is the ratio of specialists who measure it from 3 or 4 [32]. In this regard, Polite and Beck presented 0.80 for the allowable lower range of CVI value [33]. An appropriate level of the accordance was determined $(\mathrm{CVI}=0.91)$ between panelists proposing that the measure of scale had a well content validity. Finally, the mean of questions for the perceptional factor was 3.22 
$\left(\mathrm{SD}^{1} 14.33, \mathrm{~N} 1 \frac{1}{4} 5\right)$, for the nurture factor was $4.3\left(\mathrm{SD}^{1} \frac{1}{4} .47, \mathrm{~N} 1 \frac{1}{4} 4\right)$ and for enabling factor was $4.3\left(\mathrm{SD}^{1} 14.47\right.$, $\mathrm{N} 1 / 44)$. All of the questions were deleted because of low grading on one of these implications.

\section{Study of statistical analysis}

A cross-validation development was exerted in that group $(n=400)$ was randomized. Data obtained from this group were employed of the scales in psychometric test development in this study. After that, the factor analysis was used to sample these dichotomous questions into scales [34]. Every factor analyses used principal components with varimax rotation. In this regard, in the scree plot, the analysis of values was employed to help assign the number of factors to preserve in a specified instrument [35]. In this context, if more than one of the factors transpired, factor analyses compressing 1, 2, and 3 factors were administered for subsequent evaluation of adequate. A question was specified to a factor when its loading was minimum $\mathbf{0 . 3 5}$, and this had no loadings at 0.30 or upper on other factors. Scores on the questionnaire scale were produced by calculating the scores of questions creating that factor. Cronbach's a was used to evaluate internal consistency in the study [36]. Finally, the test-retest reliability was specified using Pearson correlations.

\section{Validity}

\section{Construct validity}

The different dimensions of the criterion were specified by implementing exploratory factor analysis (EFA) to apply the oblique rotation and original fundamental factoring. As the correlation between factors was less than 0.3, varimax rotation with Kaiser Normalization was chosen [37]. Because of assessment, sampling efficiency to carry out the satisfactory factor analysis, Kaiser-Meyer Olkin (KMO) Measure of Sampling Adequacy (MSA) for factor analysis and Bartlett's test was accounted for. To specify the supreme construction, the specific value more significant than one and factor loading equivalent to or larger than 0.4 were used [38].

\section{Convergent validity}

Validity of convergent was determined to implement correlations of item-scale that reformed for overlaps. In this part, the correlations were computed, applying a Pearson's correlation coefficient. It was anticipated that scores of the item would correlate greater with a specific assumed scale than other scales. The values of correlation of 0.40 or higher were investigated satisfying $(r \geq 0.81-1$ as supreme and excellent, $0.61-0.80$ very good, $0.41-0.60$ good, $0.21-0.40$ fair, and 00-0.20 poor) [39].

\section{Reliability}

\section{Test-retest}

In this study, the test-retest reliability test was evaluated by an intraclass correlation coefficient (ICC). The criterion was re-managed to 45 parsons one month since the first supplementation. The following 
classification was chosen to construe the compromise levels: 00-0.2 as low, 0.21-0.40 as fair, 0.41-0.60 as moderate, $0.61-0.80$ as significant, and $0.81-1$ as nearly complete and perfect [39].

\section{Internal consistency}

In this study, the internal consistency (IC) of PA2Q-PW accounted for by calculating Cronbachs' alpha coefficient. The alpha amount of 0.70 or higher was noticed satisfactory [39].

\section{Scoring}

The last version of the PA2Q-PW is present. Additional information about this questionnaire and the type of scoring is also available. Nevertheless, in brief scoring, each of these items is from 5 to 1 to create row scores. Like, two of these items on nurtures problem, and susceptibility of doing exercise, and others concerning one's stimulus were nugatory pointed, scoring toward those items (items 28 , in the first version and 31 in the last version) was converted.

\section{Ethical considerations}

The Ethics Committee approved the study of Tarbiat Modares University. All of the pregnant women, as participants, confirmed their permission by signing an aware consent form.

\section{Consent to participate}

To participate in the human survey, all the pregnant women's written satisfaction was taken, and each time they wished to withdraw from the study with their consent. All of the records data in this study were preserved private in the match with organizational policies on topic solitude. The researcher and other site staff will not utilize such records and information for any target other than operating the survey. In terms of confidentiality, pregnant women and their husbands were told at the beginning of each assessment that their responses are confidential and that they will not have access to each other's answers.

\section{Consent for publication}

The consequence of this trial will be broadcasted through peer that reviewed journal articles, introductions at newsletters about pregnant women, and international conferences to be held. All participants gave informed written consent.

\section{Results}

Everywhere 400 pregnant women engaged in this trial. The average age of women was $32.40(\mathrm{SD}= \pm$ 11.4 ) years. The first gravid ladies $(86 \%)$ read until secondary school and had no exercise within gestation. The findings are shown in Table2. In this way, exploratory factor analysis (EFA) was exerted to determine the fundamental factor structure of the collection of items. The assessed KMO was 0.73 , and Bartlett's test for Sphericity was considerable $(P<0.0001)$. A three-factor settlement, together with 39 
items, transpired based on specific values greater than one and a loading level of 0.4 or higher. The three factors were titled due to the fundamental construct that pertinent to the following items: operational perception nurtures and enabling. The three-factor settlement described $78.30 \%$ of all collected variances from the assumed model. The detailed findings are presented in Table 3. Interpretive census containing mean scores, intra-class correlation coefficient (ICC), Cronbach's alpha, and deviations for the PA2Q-PW are shown in Table 4. The value of Cronbach's alpha for the subscales averaged from 0.70 to 0.88 . The subscales value of the intra-class correlation coefficient (ICC) for the PA2Q-PW was hopeful (ICC averaged from 0.70 to 0.92 ). As anticipated, the correlation between total items, depending on the constructs of the Theory of PEN-3 cultural model was satisfactory.

Convincing (ICC average of 0.71 to 0.92 ). In this paper, the correlation matrix is reported in Table 6 . As recognized, the correlation among segments depending on all of the constructs of the PEN-3 cultural model was satisfactory.

\section{Scale refinement}

In this study, analyses were operated with the 39 items introduced by skillful experts in the primary phase of scale design. Firstly, we inspected every changeable dispensation based on presented manners (40) and distinguished that there were no complicated or awkward items. A principal component analysis (PCA) and exploratory factor analysis sans rotation were so guided (41). The findings scree plot displayed a three-factor resolution. Subsequent, perform an oblimin rotation with principal component analysis (PCA) was guided determined the attended factors versus correlation. Nevertheless, the finding principal components of the correlation (covariance) matrices demonstrated no correlation among the three factors (correlation coefficients averaged amongst 0.19 and 0.13 ).

After that, we did a principal component analysis along with forcing three of the factors and varimax rotation, determined the deficiency of correlation among these factors. An interplayed stage was used for items with supreme cross-loadings or low loadings on these factors (no factor loadings Z.45) were deleted, and again the factor analysis was a rerun. A cut off factor loading value of .45 is a little superior to the proposed cut off .40 (42) that we select because support diminishes the last number of questionnaire items for any subscale. In to. Overall, two items were deleted in this procedure because of low cross-loadings or high factor loading as well as, correlations of inter-item were afterward inspected for every factor until recognizing a high level of redundant items, According to the criteria of numerous correlations of inter-item above $.5(40)$.

Preliminary analyses demonstrated which the second factor accomplished weakly due to constructing validity (i.e., Losing to correlate, and in several files correlating in a contrary way, with significantly relevant constructs) and was thus deprived of subsequent analyses. In this study, Principle component analysis along varimax rotation was guided with the 39 items of factors 2 , compressing three factors. The findings illustrated that the same schema as prior analyses along the 39 items loading towards the same three factors predesignate over. Eventually, three of factor resolution computed so that $40.5 \%$ of the beholden variance. 
The remaining 39 items any loaded towards one of two distinguished factors, along with minimum crossloading. In this case, Factor 1 included 27 items evaluating perceptions (e.g., the knowledge to act physical activity during pregnancy, attitude about exercise during pregnancy). Instance items for Factor 1 as follows "Exercise during pregnancy can lead to reduced oxygen supply to the baby." In addition, "I believe exercising during pregnancy reduces my fatigue due to my pregnancy." Factor 2 contained six items assessing nurtures with physical activity (e.g., Social support thoughts, encouragement for the act). Factor 3 contained six items assessing enabling with physical activity (e.g., Facilities for physical activity in pregnancy).

\section{Reliability}

Among available pregnant women, 400 pregnant women were selected for ICC (intra-class correlation coefficient), Cronbach's alpha, and 45 pregnant women were selected for the test-retest procedure on two separate dates. The intra-class correlation coefficient of the four self-reported subscales of the PA2Q were 0.90 to 0.98 , while the Cronbach's alpha coefficients from all subscales were high, varying range from 0.89 to 0.97 , alpha for all items was $0.93(\mathrm{~N}=400)$, which indicated the excellent internal consistency and test-retest reliability from the desired sections. Table 5 represents Cronbach's alpha (coefficient alpha) and intra-class correlation from the items inter-rater agreement.

\section{Construct validity}

Due to the nature and difficulty of the physical activity assessment, we had to measure fewer pregnant women than other subscales for construct validity $(\mathrm{N}=200)$, so it was analyzed separately (showed in Table 6). The confirmatory factor analysis results for the four sections of the PA2Q showed a $\chi^{2}$ value at 3921.78; degrees of freedom 1117, which gave a ratio $\chi^{2} / \mathrm{dF}=3.51(\mathrm{P}<0.0001)$. The Value of the CFI, NFI and GFI respectively, were $0.90,0.80$ and $0.92(P<0.0001)$. With regard to the RMSEA, the value was $0.091(P<0.0001)$. Figs. 1 shows the factor weighting value results for the four Knowledge, Attitude, Nurtures, and enabling subscales in the standard estimation mode.

\section{Factor Analysis}

\section{Perceptions}

The perception scale contained two factors (knowledge and attitude). In the knowledge factor, total items loaded a minimum of 0.40 in scale (Table 7) and in for attitude factor total items loaded a minimum of 0.50 (Table 8 ). In this part of the knowledge factor, the internal consistency reliability was 0.87 in the sample $(n=400)$ as well as, the internal consistency reliability for attitude factor was 0.70 in the development sample. The correlation coefficient assessed the test-retest reliability, and for knowledge, the factor was estimated 0.97 and for attitude factor was 0.99 .

\section{Nurtures factor}


This scale contained six items. All the burden of the item average was 0.38 (Table 9). In this questionnaire, the internal consistency reliabilities about the nurtures scales were averaging 0.86 in the progress sample $(n=400)$. In this way, the test-retest reliability of the nurtures criterion was approximately 0.98 .

\section{Enabling factor}

This factor included six items in this questionnaire. Whereas six items transpired perusing the scree plot analysis. The factors were labeled, enabling factors (showed Table 10). For the enabling factors in this study, the internal consistency reliabilities were approximately $0.71(n=400)$. For this item, the test-retest correlation coefficients were at least 0.98 .

Table 11 also demonstrates fitness indicators for the four parts of the PA2Q. As may be seen, the Comparative Fit Index, goodness-of-fit ( $\chi^{2} / \mathrm{dF}$ ratio), and the other indexes (RMSEA \& GFI values) where a reasonable value, and confirmatory factor analysis results indicate that the items of the PA2Q were verified; therefore, CFA is an effective procedure to construct validity.

\section{Discussion}

This study aimed to design a criterion for evaluating pregnant women's perceptions concerning physical activity and investigating psychometric attributes and properties. This criterion was designed according to the Theory of the PEN-3 cultural model framework. In the PEN-3 cultural model, every predictive determinant could be measured straightly, e.g., By investigating participants about all over their tendency, or indirectly, e.g., By investigating participants about particular behavioral perceptions, nurtures, and enabling factors about physical activity during pregnancy. Commonly for designing and developing a questionnaire based on the PEN-3 cultural model, it has been proposed that for direct scales, one could use the similar direct scales designed by Airhihenbuwa [37]. Regarding indirect (based on perceptions) scales; it has been suggested to accomplish an exploitation study to design all of the constructs about predictive factors in the PEN-3 that consist of knowledge, attitude, nurture, and enabling. In reality, in this research, various assumptions create due to direct and indirect views about the fundamental cognitive structures, and neither view is complete. When several procedures are knapping a similar construct, degrees are anticipated to be affirmatively associated, so it is suggested that both of them be contained of a questionnaire with PEN-based [37]. Unfortunately, just only direct scores of the PEN-3 cultural model theory-based were used in this study.

Perception, nurture, and enabling that perception contained two subscales (knowledge and attitude). In this way, exploratory factor analysis was made to eliminate two items of the questionnaire from a whole of the essential 41 items and 39 scales classified into three agents, clarifying $70.3 \%$ of the variance. In this study, varimax rotation with EFA illustrated that three agents containing perception, nurture, and enabling can be earned. Two subscales were derived from perceptions factors (knowledge, attitude) as 
expected; however, the enabling and nurture factor did burden as unique factors, and anticipated subscales separately were not transpired.

There are several practicable significations for expanding a criterion to extent pregnant women's perceptions into arranged exercise during pregnancy. Instruments for validity and reliability the questionnaire are required for developing and appraising health programs funded by behavior alteration theories of subjectivity and basic sciences [38]. In this study, interventions that were done to increase arranged exercise may purpose the opinions or beliefs that lead to behavior. To be more precise and clear, recognizing perceptions, nurtures, and enabling factors could conduct to design exciting plans and probably assist in attaining a healthy lifestyle for purpose societies. People who work with pregnant women, such as health care providers, can use the scales to recognize perceptions, nurture and enable factors that increase arranged exercise and, in the same way, interfere to postpone or prevent possible outcomes. The PA2Q-PW, as well as could be a beneficial instrument for appraising the effects of intervention by measuring the scales before and after pregnant women taking several interventions in this period, also for illustrating the correlations of perceptions, nurtures, and enabling factors of women with their exercise behavior.

For the perceptions, nurtures, and enabling factors, the internal consistency of reliabilities was above 0.80 in the development sample. For the perceptions, nurtures, and enabling factors, the test-retest correlation coefficients were about 0.90 in the Intended sample. These rates are checked sufficiently [39]. Regarding the attitude scale, the correlation coefficients and internal consistency for reliability were slightly mitigating.

This survey used a changeable approach more acceptable for pregnant women. As well as the internal consistency of reliabilities were higher than those received in prior function with first pregnant women that contained perceptions toward consequences [43]. The internal consistency reliability of attitude about the purpose of being exercised (0.63) was sufficient, however not broad. However, it is higher than the test-retest reliability presented in previous work for pregnant women [43]. It should, too, be distinguished that the reliability for the retest-retest in this survey was managed immediately after the first data collection. In this study, the ICC for total scale about physical activity was 0.92 , and it shows higher than was used questionnaire those obtained in previous work with pregnant women [43].

This survey's further contributions were used to validate the questionnaire construct and Chartered Financial Analyst (CFA) method, which can be exerted to trial numerous variables while there is a theoretical framework [44]. Moreover, various indicators, such as $\chi^{2} / \mathrm{dF}$ ratio, $\mathrm{CFI}, \mathrm{GFI}$, and RMSEA, verified the compatibility of the models. The results regarding the (NFI, RMSEA, and CFI) were higher than expected. Additionally, this finding demonstrated that each of the four sub-constructs in the PA2Q had an appropriate fit within the PEN-3 cultural model framework.

Empirical results from the Cronbach's Alpha, test-retest, and interrater reliability confirmed that, within the four sub-constructs, the PA2Q shows acceptable internal consistency (ranged from 0.92 to 0.97); provides 
reliable results over repeated administrations (ranged from 0.97 to 0.99 ) at the pregnant women during pregnancy. In previous studies, the questionnaires' reliability was evaluated just from the perspective of test-retest stability and internal consistency. For example, Satter et al. (2018) evaluated different instruments based on previous literature. Reliability indicates it ranged from 0.70 to 0.80 (45). ICC reliability of the quality evidence was 0.70 , and the other our intended section not applicable. The recent physical activity questionnaire (RPAQ) demonstrated an adequate correlation with the accelerometer that used for physical activity assessments for whole times for being active $(r \geq 0.50)$ but not for all of the physical activity energy expenditure (PAEE) and other approximations of physical activity [43]. The findings extracted from studies from the validity of these construct of the pregnancy, physical activity questionnaire (PPAQ) was mostly inadequate, like that for the Vietnamese versions of the questionnaire [46]. Chasan-Taber et al., Who designed the questionnaire, managed the pregnancy, physical activity questionnaire (PPAQ) to 54 women with the first pregnancy aged 16 to 40 to evaluate its two-week reliability and determined ICCs 0.78 for total, sedentary [47].

Therefore, according to the authors, the PA2Q is suitable for a variety of potential applications to measure physical activity and its main determinants among pregnant women. Besides, the PA2Q tool may be used with other grade populations and may be suitable for Non-pregnant women. One unique feature of PA2Q is the reliability and validity of its subscales, which include knowledge and attitude, nurtures, enabling, and physical activity behavior. These sub-constructs may be measured, evaluated, modified by potential change strategies, thereby doing physical activity during pregnancy and, finally, health promotion.

\section{Limitations}

In this study, some limitations must be noted. First, data were only collected from the two and threetrimester pregnant women attending health centers in capital Tehran's region 5; and other independent health centers, other gestational ages during pregnancy did not enroll to study; So, the generalizability of results to all of the society may be confined. Due to decreased recall bias, the physical activity report was limited within the last week. Subscales of the PA2Q were limited to the main Determinants of Behavior in the PEN-3 cultural model and the other constructs (Psychological Determinants of Behavior), in other to decrease the questions' burden on participants, did not use. In the nurture items construct validity verification phase, the sample was limited to pregnant women and no their families because of the difficulty of assessing.

Notwithstanding the limitations that have been explained, the PA2Q is the instrument of validity and reliability to assess women's exercise behavior through pregnancy. In this study with these results must be interpreted with precaution. This survey had various restrictions: only married women were considered in this study. Likewise, only direct criteria and scales were considered in this study. The future studies that applying this questionnaire could assist and help to overcome this kind of problem.

\section{Conclusion}


The goal of this study and paper was to design, development, and assessment questionnaire of the PA2Q for the pregnant women and psychometric testing of physical activity behavior and its main determinants. From the results of our investigation, it is possible to conclude that the PA2Q-PW is the first standard tool based on the PEN-3 cultural model that researchers can utilize to gather data and conduct the desired education interventions to change physical activity behavior in the pregnant women during pregnancy. The PA2Q-PW was demonstrated to be consistent and reliable within its sub-constructs of knowledge, attitude, nurtures, enabling, and behavior. However, future attempts are carried to assess whether PA2Q-PW is applicable in the first trimester of pregnancy in pregnant women populations attending a variety of community-based settings, including the health services center. Throughout, this study's results offer that the P2AQ-PW is an instrument that has been validated, and reliability is done; therefore, it can be used to assess perceptions, nurtures, and enabling factors of pregnant women concerning physical activity during pregnancy. The results of this study confirmed Airhihenbuwa's PEN-3 cultural model. Future studies are suggested to support its application in health care centers and clinics.

\section{Abbreviations}

LMICs: Low- and middle- income countries; SES: Socioeconomic status; GDM: Gestational diabetes mellitus; ACOG: American college of obstetrics and gynecology; QOL: quality of life; WHO: World Health Organization

\section{Declarations}

\section{Ethics approval and consent to participate}

The Research Ethics Committee approved the research of this study. All pregnant women in this trial were informed about participating in the survey and gave written informed consent for the study.

\section{Consent for publication}

Not applicable.

\section{Availability of data and materials}

All data generated during the process of this research are included in this article.

\section{Funding}

This study is funded in part by the Medical Sciences, Tarbiat Modares University, Department of Health Education and Health Promotion. This funding has facilitated the use of websites to support physical activity during pregnancy.

\section{Competing interests}


The authors declare that they have no competing.

\section{Authors' contributions}

LK comprehended the trial, completed the collection, categorized the data. SR presented supervise during data collection and collaborated to temporary and interpretation final data. SHN performed manage during the development of the study and data collection, participated in the analysis of the data. FSH prepared the draft, and edited the repetitive draft. All researchers read, concluded, and approved the final draft.

\section{Acknowledgements}

We gratefully thank all the pregnant women, their families, and health centers who accompanied us along with this study. We would also like to thank the research deputy of Tarbiat Modares University for its financial support. This study was a part of the doctoral dissertation of the first author in health education and promotion at the Faculty of Medical Sciences, Tarbiat Modares University. It was approved by the university's institutional review board (IRB) on August 18, 2018. We wish to acknowledge the assistance and support of authorities and faculty members in the Faculty of Medicine and Tarbiat Modarres University. The authors would like to extend their thanks to the Ministry of Education and District five authorities and staff in Tehran for implementing the project.

\section{References}

1. World Health Organization. World health statistics, 2010. http://www.who.int/whosis/whostat/ 2010/en/. Accessed 3 October 2015.

2. Warburton DE, Nicol CW, Bredin SS. Health benefits of physical activity: the evidence. Can Med Assoc J. 2006;174(6):801-9.

3. Dempsey JC, Sorensen TK, Williams MA, et al. A prospective study of gestational diabetes mellitus risk concerning physical activity before and during pregnancy. Am J Obstet Gynecol. 2003;189(6):S106.

4. Kieffer EC, Willis SK, Arellano N, Guzman R. Perspectives of pregnant and postpartum Latino women on diabetes, physical activity, and health. Health Educ Behav. 2002;29(5):542-56.

5. Shisana O. The South African National Health and Nutrition Examination Survey: SANHANES-1. HSRC Press, 2013. http://www.hsrc.za/uploads/ pageNew/72/SANHANES Sliaunch \%20 edition \%20(online\%20 version).Pdf. Accessed 14 November 2015.

6. Artal R, O'Toole M. Guidelines of the American College of Obstetricians and Gynecologists for exercise during pregnancy and the postpartum period. Br J Sports Med. 2003;37(1):6-12.

7. American College of Obstetricians and Gynecologists. Exercise during pregnancy and the postpartum period. Clin Obstet Gynecol. 2003;46(2):496 9. 
8. Gynecologists ACoOa. ACOG Committee Opinion. Number 267, January 2002: exercise during pregnancy and the postpartum period. Obstet Gynecol. 2002;99(1):171-3.

9. Artal R, Catanzaro R, Gavard J, Mostello D, Friganza J. A lifestyle intervention of weight-gain restriction: diet and exercise in obese women with gestational diabetes mellitus. Appl Physiol Nutr Metab. 2007;32(3):596-601.

10. Sui Z, Grivell R, Dodd J. Antenatal exercise to improve outcomes in overweight or obese women: a systematic review. Acta Obstet Gynecol. 2011;91:538-45.

11. Nascimento S, Surita F, Cecatti J. Physical exercise during pregnancy: a systematic review. Curr Opin Obstet Gynecol. 2012;24:387-94.

12. Esmaelzadeh S, Taavaoni S, Ahmadi Z, Haghani H. Trend of exercise before, during, and after pregnancy. Iran J Nurs. 2008;21:135-41.

13. 13. Ghafoori FS, Rostami NM, Nasrolahi A, Darabi S, Sokhtezari S. Evaluating the association between quality of life and physical activity in female student children scientific. J Ilam Univ Med Sci. 2013;21:144-51

14. Mirghafourvand $M$, Mohammad-Alizadeh Charandabi S, Asghari Jafarabadi M. Mohammadi A, Soltanpour Gharibdoosti S. The Relationship between Physical Activity during Pregnancy and Postpartum Mood in Primiparous Women. J Babol Univ Med Sci. 2016;18:35-41.

15. Mparmpakas D, Goumenou A, Zachariades E, Pados G, Gidron Y, KarterisE. Immune system function, stress, exercise, and nutrition profile can affect pregnancy outcome: Lessons from a Mediterranean cohort. Exp Ther Med J. 2013;5:411-418.

16. Godin G, Shephard RJ. Use of attitude-behavior models in exercise promotion. Sports Med. 1990;10:103-21.

17. Iwelunmor J, Newsome $V$, Airhihenbuwa CO. Framing the impact of culture on health: a systematic review of the PEN 3 cultural model and its application in public health research and interventions. Ethn Health. 2014;19(1):20-46.

18. Sallis JF, Hovell MF, Hofstetter CR. Predictors of adoption and maintenance of vigorous physical activity in men and women. Prev Med. 1992;21:237-51.

19. Reynolds KD, Killen JD, Bryson SW, Maron DJ, Taylor CB, Mac- Coby N, Farquhar JW. Psychosocial predictors of physical. Prev Med. 1990;19(5):541-51.

20. Sallis JF, Simons-Morton BG, Stone EF, et al. Determinants of physical activity and interventions in youth. Med Sci Sports Exerc. 1992;24:S248-57.

21. Ussher $M$, Lewis $S$, Aveyard $P$, et al. Physical activity for smoking cessation in pregnancy: a randomized controlled trial. BMJ. 2015;14:350.

22. Hamilton K, White KM. Extending the Theory of planned behavior: the role of self and social influences in predicting adolescent regular moderate-to-vigorous physical activity. J Sport Exerc Psychol. 2008; 30:56-74. 
23. Sparks P, Guthrie CA. Self-Identity and the Theory of planned behavior: A useful addition or an unhelpful artifice? J Applied Social Psychol. 1998;28:1393-410.

24. Harrod CS, Chasan-Taber L, Reynolds RM. Physical activity pregnancy and neonatal body composition: the Healthy Start study. Obstetrics \& Gynecology. 2014;124:257-264.

25. Esmaelzadeh S, Taavaoni S, Ahmadi Z, Haghani H. Trend of exercise before, during, and after pregnancy. Iran J Nurs. 2008;21:135-41.

26. Ghafoori FS, Rostami NM, Nasrolahi A, Darabi S, Sokhtezari S. Evaluating the association between quality of life and physical activity in female student children scientific. J Ilam Univ Med Sci. 2013;21:144-51.

27. Sarrafzadegan N, Boshtam M, Rafiei M: Risk factors for coronary artery diseases in Isfahan. Eur J Public Health. 1999;1:20-6.

28. Kelishadi R, Sadri G, Tavasoli AA, et al. Cumulative prevalence of risk factors for atherosclerotic cardiovascular diseases in Iranian adolescents: IHHP-HHPC. J Pediatr. 2005;81:447-53.

29. Sarrafzadegan N, Kelishadi R, Baghaei A, et al. Metabolic syndrome: an emerging public health problem in Iranian women: Isfahan Healthy Heart Program. Int J Cardiol. 2008;131:90-6.

30. Chasan-Taber L, Schmidt MD, Roberts DE, et al. Development and validation of a Pregnancy Physical Activity Questionnaire. PubMed Sci Sports Exerc.2004;36(10):1750-60.

31. Lawshe CH. A quantitative approach to content validity. Personal Psychol. 1975;28:563-75.

32. Grant JS, Davis LL. Focus on quantitative methods: selection and use of content experts for instrument development. Res Nurs Health. 1997;20:269-74.

33. Polit DF, Beck, CT. Nursing research: principles and methods. 46 ed. Philadelphia: Lippincott; 2004.

34. Kim J-O, Mueller CW. Factor analysis: statistical methods and practical issues. Sage University paper, 1987.

35. Cattell RB. Factor analysis: an introduction to essentials. I. The purpose and underlying models. II. The role of actor analysis in research. Biometrics. 1965;21:190-215.

36. Carmines EG, Zeller RA. Reliability and validity assessment. Sage University paper, 1979.

37. Iwelunmor J, Newsome V, Airhihenbuwa CA. Framing the impact of culture on health: a systematic review of the PEN-3 cultural model and its application in public health research and interventions. Ethnicity Health 2014;19(27): 20-46.

38. Blue CL, Marrero DG. Psychometric properties of the healthful eating belief scales for persons at risk of diabetes. J Nutr Educ Behav. 2006;38:1341-42.

39. Nunnally JC. Psychometric Theory, 2nd ed. McGraw-Hill Book Co., 1978.

40. 40. Antunes $R$, Couto $N$, Vitorino $V$, et al. Physical activity and affect of the elderly: Contribution to the validation of the Positive and Negative Affect Shedule (PANAS) in the Portuguese population. Journal of Human Sport and Exercise. 2020;15:2-8

41. Maskey R, Fei J, Nguyen $\mathrm{H}$. Use of exploratory factor analysis in maritime research. The Asian Journal of Shipping and Logistics.2018; 34:91-111 
42. Floyd, F. J, Widaman, K. F. Factor analysis in the development and refinement of clinical assessment instruments. Psychological Assessment. 1995; 7: 286-299.

43. Çırak Y, Yılmaz GD, Demir YP, Dalkılınç M, Yaman S. Pregnancy physical activity questionnaire (PPAQ): reliability and validity of Turkish version. J Phys The Sci. 2015;27: 3703-3709.

44. Vasli P. Translation, Cross-Cultural Adaptation, and Psychometric Testing of Perception of FamilyCentered Care Measurement Questionnaires in the Hospitalized Children in Iran. J Pediatr Nurs [Internet]. Elsevier Inc. 2018; 43: 26-34.

45. Sattler MC, Jaunig J, Watson ED, et al. Physical Activity Questionnaires for Pregnancy: A Systematic Review of Measurement Properties. Sports Med.2018; 48(10):2317-2346.

46. Ota E, Haruna M, Yanai H, Suzuki M, et al. Reliability and validity of the Vietnamese version of the Pregnancy Physical Activity Questionnaire (PPAQ). Southeast Asian J Trop Med Public Health.2008;39(3):562-70.

47. Chasan-Taber L, Schmidt MD, Roberts DE, et al. Development and validation of a Pregnancy Physical Activity Questionnaire. Med Sci Sports Exerc. 2004;36:1750-1760.

\section{Tables}

Due to technical limitations, table 1 docx is only available as a download in the Supplemental Files section.

Table 2. Medical and Demographic Profile of the pregnant women $(n=400)$ 
No. (\%)

\begin{tabular}{|c|c|c|}
\hline Age & Year, Mean (SD) & $32.40 \pm 12.40$ \\
\hline Gestational Age & Week & $264 / 7 \pm 6 / 7$ \\
\hline \multicolumn{3}{|l|}{ Education } \\
\hline & Elementary & $93(20)$ \\
\hline & Middle & $253(62)$ \\
\hline & Higher & $54(12)$ \\
\hline \multicolumn{3}{|l|}{ Economic status } \\
\hline & Low income & $242(60)$ \\
\hline & Middle income & $76(19)$ \\
\hline & High income & $82(20.5)$ \\
\hline \multicolumn{3}{|l|}{ Employment } \\
\hline & Housekeeper & $232(58)$ \\
\hline & Employed & $148(37)$ \\
\hline & Student & $20(5)$ \\
\hline$\overline{\mathrm{BMI}}$ & Mean (SD) & $26.80 \pm 6$ \\
\hline Duration & Year, Mean (SD) & $9.50 \pm 5$ \\
\hline
\end{tabular}

Table 3. Findings received from the exploratory factor analysis (EFA). 


\begin{tabular}{|c|c|c|c|c|}
\hline Items & F1 & $\mathrm{F} 2$ & F3 & F4 \\
\hline 1 During pregnancy, you should not exercise ..... & .95 & .55 & -.036 & .001 \\
\hline 2 Physical activity is essential to prevent ... & .79 & .056 & -.043 & -.21 \\
\hline 3 Exercise during pregnancy can lead to reduced oxygen.... & .45 & .056 & -.055 & -.11 \\
\hline 4 During pregnancy, you should exercise smoothly ..... & 48 & -.026 & .038 & .056 \\
\hline 5 Pregnant women during exercise should avoid excessively ... & 94 & .28 & .014 & .006 \\
\hline 6 Exercise during pregnancy makes it easy for normal labor. & .94 & .32 & .024 & -.005 \\
\hline $\begin{array}{l}7 \text { Exercise in pregnancy reduces the risk of birth in a diabetic } \\
\text { neonatal. }\end{array}$ & .78 & .22 & .35 & -.036 \\
\hline 8 Doing basic exercises daily will not hurt the mother and baby. & 45 & .26 & .038 & .022 \\
\hline 9 Exercise eliminates the risk of pregnancy complications ... & .55 & .02 & -.055 & 0.28 \\
\hline 10 During pregnancy, you should stop ... & .84 & 0.15 & 0.01 & -0.03 \\
\hline 11 Before starting exercise during pregnancy, you should... & .74 & 0.09 & 0.18 & -0.04 \\
\hline 12 Stretching and strength during pregnancy should be ... & .45 & .11 & -.033 & -.45 \\
\hline 13 Exercising in pregnancy prevents blood pressure in danger. & .93 & .15 & -.01 & .01 \\
\hline 14 Exercise during pregnancy can lead to a return... & .92 & -.025 & -.027 & .00 \\
\hline 15 I try to avoid lifting any weight during pregnancy... & .65 & .053 & .040 & -.1 \\
\hline 16 Exercising during pregnancy leads to fitness .... & .97 & .27 & -.030 & .01 \\
\hline 17 For pre-exercise preparation, 15 minutes of relaxation... & .85 & .64 & .33 & -.044 \\
\hline 18 Before conducting any exercise at this time.... & .43 & .02 & -.057 & .03 \\
\hline 19 Exercise reduces the risk of musculoskeletal discomfort. & .90 & .20 & -.016 & .02 \\
\hline 20 In the third trimester of pregnancy, the intensity .... & .93 & -.030 & -.070 & -.01 \\
\hline 21 I believe that with exercise, I can ... & .21 & .85 & -.055 & .03 \\
\hline 22 I believe exercising during pregnancy reduces... & -.065 & .89 & -.011 & .03 \\
\hline 23 I believe that I can work out stress ... & -.013 & .94 & .001 & .07 \\
\hline 24 I believe exercising during pregnancy helps... & -.05 & .91 & .42 & .02 \\
\hline 25 I believe that I can easily maintain my fitness... & .001 & .46 & .002 & .01 \\
\hline 26 I believe that by exercising, I can reduce it... & -.01 & .93 & .024 & .09 \\
\hline 27 I believe that if I exercise in pregnancy I can... & .06 & .48 & -.118 & .85 \\
\hline 28 If my wife is in physical education classes ... & .24 & -.038 & .052 & -.08 \\
\hline
\end{tabular}




\begin{tabular}{|l|rrrr|}
\hline 29 If my own family and my wife attend ... & .34 & -.036 & .78 & .09 \\
\hline 30 I encourage friends and acquaintances to ... & .06 & -.012 & .83 & .03 \\
\hline 31 If after a pregnancy class, there is a group ... & .01 & .027 & .90 & .00 \\
\hline 32 If, after a pregnancy class, there is a skilled and ... & -.02 & -.057 & .45 & -.03 \\
\hline 33 If my doctor advises me to do exercise ... & .38 & -.016 & .67 & .09 \\
\hline $34 \quad$ If there is a good place to exercise... & .01 & -.024 & .10 & .86 \\
\hline 35 If classes are held free, I can use them... & .00 & -.016 & .02 & .86 \\
\hline $36 \quad$ If it is a physical activity area near ... & -.03 & -.032 & .01 & .88 \\
\hline $37 \quad$ If I have an educational CD in the centers... & .05 & -0.14 & .26 & .48 \\
\hline 38 If there are enough facilities, such as... & .32 & 0.06 & 0.27 & .60 \\
\hline 39 If exercise classes take place at different ... & .01 & -.02 & .08 & .43 \\
\hline
\end{tabular}

F1: Knowledge, F2: Attitude, F3: Nurtures, F4: Enabling

Table 4. Descriptive statistics, Intra-class correlation coefficient (ICC) and Cronbach's alpha of the PA2QPW sub-constructs.

\begin{tabular}{|llllll|}
\hline & No of items & Mean $(\mathrm{SD}) *$ & Skewness & Cronbach' alpha & ICC** \\
\hline Knowledge & 20 & $86.80(12.80)$ & -1.71 & 0.96 & 0.76 \\
\hline Attitude & 7 & $78.50(14.70)$ & -0.81 & 0.97 & 0.79 \\
\hline Nurture & 6 & $75.40(16.50)$ & -0.70 & 0.94 & 0.83 \\
\hline Enabling & 6 & $58.10(25.70)$ & -0.40 & 0.89 & 0.89 \\
\hline
\end{tabular}

*Scores average from 0 to 100 . Scores higher than that illustrate better conceptions concerning physical activity.

**Intra-class Correlation Coefficient

Table 5. The ICC \& Alpha of the PA2Q subscales. 


\begin{tabular}{|llll|}
\hline Subscale & ICC $(\mathrm{N}=45)$ & P Value & $\mathrm{a}(\mathrm{N}=400)$ \\
\hline Knowledge & 0.96 & 0.000 & 0.96 \\
\hline Attitude & 0.90 & 0.000 & 0.97 \\
\hline Nurtures & 0.94 & 0.000 & 0.94 \\
\hline Enabling & 0.97 & 0.000 & 0.89 \\
\hline Behavior & 0.98 & 0.000 & 0.95 \\
\hline Total & 0.84 & 0.000 & 0.93 \\
\hline
\end{tabular}

Statistical significance p-value $<0.05$.

Table 6. Report of the panelists rating for four sub-scales CVR \& CVI (N=19). 


\begin{tabular}{|c|c|c|}
\hline Variable \& Items & CVR (\%) & CVI (\%) \\
\hline \multicolumn{3}{|l|}{ Knowledge } \\
\hline During pregnancy, you should not exercise... & 84 & 100 \\
\hline Physical activity is essential to..... & 78 & 92 \\
\hline Exercise during pregnancy can lead ..... & 100 & 100 \\
\hline During pregnancy, you should exercise..... & 84 & 88 \\
\hline Pregnant women during exercise should ..... & 78 & 82 \\
\hline Exercise during pregnancy makes..... & 84 & 100 \\
\hline Doing basic exercises will not..... & 82 & 88 \\
\hline Exercise eliminates the risk of pregnancy..... & 78 & 88 \\
\hline During pregnancy, you should stop ..... & 82 & 92 \\
\hline Before starting exercise during pregnancy.... & 88 & 100 \\
\hline Stretching and strength during pregnancy ..... & 92 & 86 \\
\hline Exercising in pregnancy prevents..... & 84 & 77 \\
\hline Exercise during pregnancy can lead to..... & 82 & 86 \\
\hline I try to avoid lifting any weight..... & 88 & 92 \\
\hline Exercising during pregnancy leads..... & 89 & 96 \\
\hline For pre-exercise preparation, 15 minutes..... & 87 & 92 \\
\hline Before conducting any exercise..... & 92 & 88 \\
\hline Exercise reduces the risk..... & 90 & 100 \\
\hline In the third trimester of pregnancy, the intensity.... & 85 & 88 \\
\hline \multicolumn{3}{|l|}{ Attitude } \\
\hline I believe that with physical activity,... & 100 & 100 \\
\hline I believe exercising during pregnancy reduces.... & 88 & 92 \\
\hline I believe that I can work out stress and anxiety..... & 74 & 88 \\
\hline I believe exercising during pregnancy..... & 84 & 95 \\
\hline I believe that I can easily maintain my fitness..... & 84 & 98 \\
\hline I believe that by exercising, I can..... & 84 & 92 \\
\hline I believe that if I exercise in pregnancy..... & 85 & 98 \\
\hline
\end{tabular}




\begin{tabular}{|lll|}
\hline Nurtures & & \\
\hline If my wife is in physical education classes.... & 85 & 100 \\
\hline If my own family and my wife attend physical education.... & 88 & 92 \\
\hline I encourage friends and acquaintances..... & 82 & 8 \\
\hline If, after a pregnancy class, there is a group... & 88 & 92 \\
\hline If my family is in physical education classes... & $52^{\star}$ & $67^{\star}$ \\
\hline If after a pregnancy class, there is a skilled..... & 90 & 100 \\
\hline If my family would advise me to do exercise .... & $32^{\star}$ & $64^{\star}$ \\
\hline If my doctor would advise me to do exercise.... & 92 & 88 \\
\hline Enabling & & \\
\hline If there is an excellent place to exercise.... & 88 & 92 \\
\hline If classes are held free, I can..... & 78 & 82 \\
\hline If it's a physical activity area near my..... & 92 & 100 \\
\hline If I have a training CD in the centers..... & 95 & 88 \\
\hline If there are enough facilities.... & 86 & 96 \\
\hline If exercise classes take place at different times.... & 86 & 92 \\
\hline
\end{tabular}

Table 7. Varimax Rotation in Principal Factor Analysis for the Social Impression Measure 


\begin{tabular}{|ll|}
\hline Knowledge & Factor \\
\hline During pregnancy, you should not exercise severe than pre-pregnancy. & 0.71 \\
\hline Physical activity is essential to prevent overweight due to pregnancy. & 0.70 \\
\hline Exercise during pregnancy can lead to reduced oxygen supply to the baby. & 0.63 \\
\hline During pregnancy, you should exercise smoothly and without sloping surfaces. & 0.62 \\
\hline Pregnant women during exercise should avoid excessive curvature and hang up. & 0.62 \\
\hline Exercise during pregnancy makes it easy for normal labor. & 0.57 \\
\hline Doing basic exercises daily will not hurt the mother and baby. & 0.41 \\
\hline Exercise eliminates the risk of possible complications in pregnancy, & 0.71 \\
\hline As. Back pain, lumbar pain, constipation, and excessive fatigue. & \\
\hline During pregnancy, you should stop doing heavy exercises. & 0.68 \\
\hline Before starting exercise during pregnancy, you should have a light stroke to warm up. & 0.56 \\
\hline A physical activity specialist should take stretching and strength during pregnancy. & 1.57 \\
\hline Exercising in pregnancy prevents blood pressure dangers. & 2.96 \\
\hline Exercise during pregnancy can lead to return faster maternal postpartum. & 1.25 \\
\hline I try to avoid lifting any weight during pregnancy. & 0.82 \\
\hline Exercising during pregnancy leads to fitness and weight control. & 0.72 \\
\hline For pre-exercise preparation, 15 minutes of gentle relaxation movements are required. & 0.67 \\
\hline Before conducting any exercise at this time, consultation with the doctor is required. & 0.58 \\
\hline Exercise reduces the risk of musculoskeletal discomfort. & 0.63 \\
\hline In the third period of pregnancy, the intensity exercise should be reduced. & 0.85 \\
\hline Percentage variance explained & 38.72 \\
\hline
\end{tabular}

Table 8. Varimax Rotation in Principal Factor Analysis for the Social Impression Measure. 


\section{Attitude}

Factor

I believe that with exercise, I can efficiently deal with problems such as gestational diabetes. $\quad 0.82$

I believe that exercising during pregnancy reduces my fatigue due to my pregnancy.

0.68

I believe that I can work out stress and anxiety from childbirth during this period.

0.56

I believe exercising during pregnancy helps with my daily activities.

1.26

I believe that I can easily maintain my fitness by exercising during pregnancy.

0.75

I believe that by exercising, I can reduce postpartum depression.

0.69

I believe that if I exercise in pregnancy, I can have a more comfortable delivery.

1.52

Percentage variance explained

Table 9. Varimax Rotation in Principal Factor Analysis for the Social Impression Measure.

\section{Nurture}

Factor

If my wife is in physical education classes in pregnancy, I can more easily convince her to attend pregnancy classes.

If my own family and my wife attend physical education classes in pregnancy, I can persuade them to participate in physical activity classes.

I encourage friends and acquaintances to exercise in pregnancy

If after a pregnancy class, there is a group discussion to exchange information about pregnant women, I have a greater tendency to attend a pregnancy class.

If after a pregnancy class, there is a skilled and informed person to answer midwifery questions, I would be more eager to participate in pregnancy classes.

If my doctor advises me to do exercise in pregnancy, I will do that.

Table 10. Varimax Rotation in Principal Factor Analysis for the Social Impression Measure. 
If there is an excellent place to exercise, it is easier to exercise in pregnancy.

If classes are held free, I can use these classes more easily.

If it is a physical activity area near my place of residence, I can get better and more comfortable with pregnancy classes.

If I have a training $\mathrm{CD}$ in the centers, I can do more exercise at home.

If there are enough facilities, such as a buffet, a washbasin and ... in pregnancy classes, I always try to take part in pregnancy classes

If exercise classes take place at different times throughout the day, I can more easily take part in physical activity classes.

Table 11. Fitness indicators of the PA2Q subscales $(\mathrm{N}=400)$.

\begin{tabular}{|llllllll|}
\hline & CFI & RMSEA & GFI & NFI & $\chi 2 /(\mathrm{df})$ & P-Value & Results \\
\hline Subscales & 0.90 & 0.091 & 0.80 & 0.92 & 3.51 & 0.000 & Confirmed \\
\hline
\end{tabular}

Statistical significance $p$-value $<0.05$.

\section{Figures}




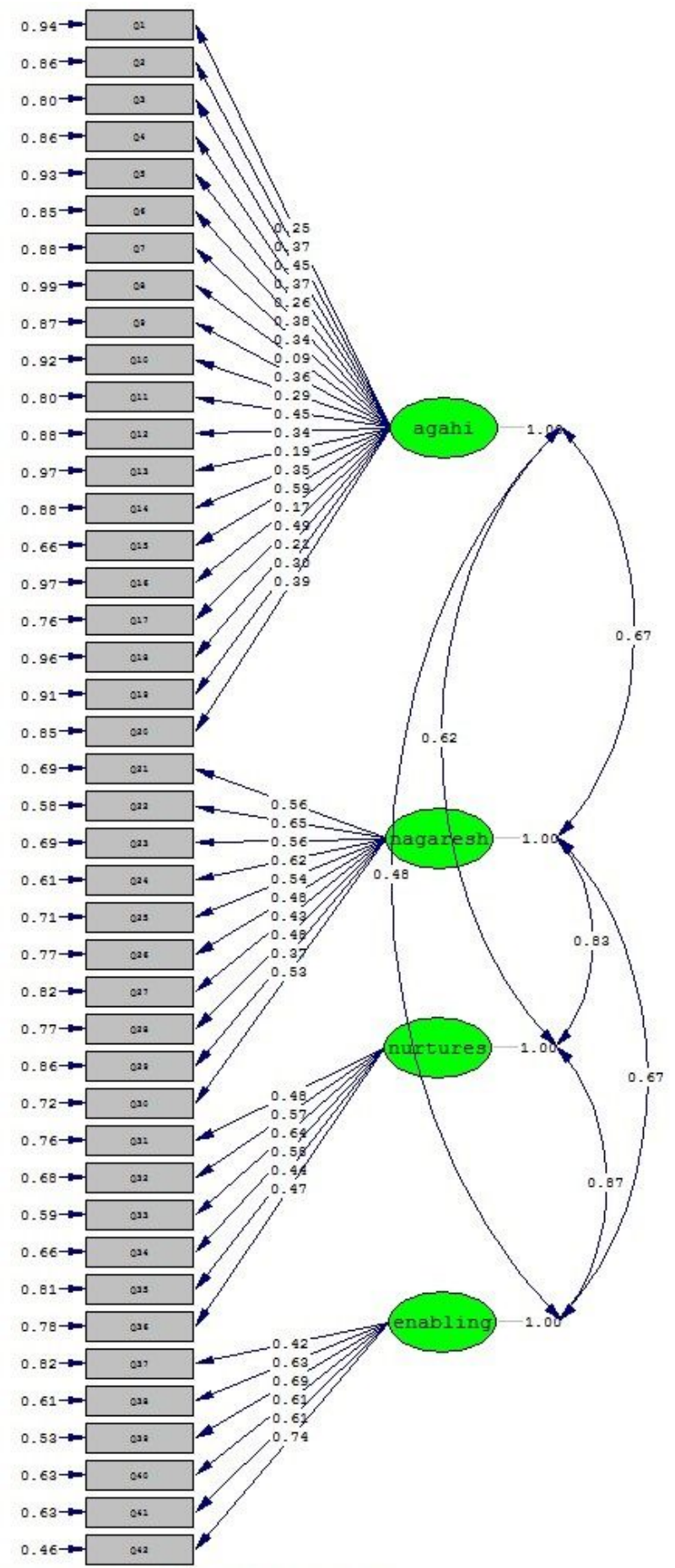

Chi-3quare $=1098.45, d f=813, p-v a 1 u e=0.00000$, RMSEA $=0.038$

\section{Figure 1}

Standard estimation model based on binary correlation coefficients of questionnaire structures

\section{Supplementary Files}

This is a list of supplementary files associated with this preprint. Click to download. 
- Table1.docx

Page 29/29 